\title{
Speech Perception in Noise Deficits in Japanese Children with Reading Difficulties: Effects of Presentation Rate
}

\author{
Tomohiro Inoue $^{1}$, Fumiko Higashibara ${ }^{2}$, Shinji Okazaki ${ }^{1}$, Hisao Maekawa ${ }^{1}$ \\ ${ }^{1}$ Department of Comprehensive Human Sciences, University of Tsukuba, Ibaraki, \\ Japan; ${ }^{2}$ Faculty of Child Studies, Seitoku University, Chiba, Japan
}

Running Head: Speech perception deficits in Japanese children with reading difficulties

Keywords: Reading difficulties; Speech perception in noise; Effects of presentation rate; Japanese

\section{Correspondence:}

Tomohiro Inoue

Department of Comprehensive Human Sciences (Disability Science)

University of Tsukuba

1-1-1 Tennodai, Tsukuba, Ibaraki, 305-8572 Japan.

Tel/Fax: +81298536760

Email: tinoue@human.tsukuba.ac.jp 


\section{Abstract}

We examined the effects of presentation rate on speech perception in noise and its relation to reading in 117 typically developing (TD) children and 10 children with reading difficulties $(\mathrm{RD})$ in Japan. Responses in a speech perception task were measured for speed, accuracy, and stability in two conditions that varied stimulus presentation rate: high rate and same rate conditions. Results showed that TD children exhibited significantly more stable responses in the high rate condition than they did in the same rate condition. Multiple regression analyses indicated that response stability in the high rate condition accounted for a unique amount of variance in reading and mora deletion. As a group, children with RD performed less accurately than did TD children in the high rate condition, but not in the same rate condition. Findings suggest that the dependence of speech perception on stimulus context relates to reading proficiency or difficulty in Japanese children. The influences of phonology and orthography of language on the relationships between speech perception and reading are discussed. 


\section{Introduction}

Developmental reading difficulty (RD), or dyslexia, is a failure to acquire age-appropriate reading skills, despite educational opportunity and at least statistically average intelligence (Snowling, 2000). Although the causal factors of these difficulties are still intensely debated, the most widely-held theory currently implicates phonological deficits (Vellutino, Fletcher, Snowling, \& Scanlon, 2004). That is, these individuals face difficulties in developing conscious awareness of segmental speech units, or manipulating them, so that they fail to map the orthographic code onto phonological code.

While phonological deficit theory is well established, others have argued that auditory processing deficits precede, and are causal of, phonological deficits. For example, it has been found that individuals with RD can exhibit problems in a variety of psychoacoustic tasks, including frequency discrimination (Banai \& Ahissar, 2004), fast temporal processing (Tallal, 1980), or amplitude modulation detection (Goswami, Thomson, Richardson, Stainthorp, Hughes, Rosen, \& Scott, 2002). In recent years, there has been additional focus on deficits in speech perception apart from general auditory processing (for a review see Rosen, 2003). Several studies have found deficits in 
individuals with RD in multiple aspects of speech perception. For instance, Adlard and Hazan (1998) found that a sub group of children with RD showed poor performance in a speech discrimination test. Similarly, McArthur, Ellis, Atkinson, and Coltheart (2008) reported that some individuals within a sample of children with RD showed abnormal thresholds in another speech discrimination task.

In addition to these findings, a number of studies found clear speech perception deficits in background noise. In the initial study of this line, Brady, Shankweiler, and Mann (1983) showed that nine-year-old poor readers performed less accurately than age-matched good readers on a repetition task requiring identification of words presented in noise. Similarly, Chermak, Vonhof, and Bendel (1989) found that adults with a learning disability consistently showed speech perception deficits in various types of noise conditions. Indeed, the majority of studies on the subject has consistently shown speech perception deficits in noise among individuals with RD in alphabetic languages as well as in nonalphabetic languages such as Japanese (Boets, Ghesquière, van Wieringen, \& Wouters, 2007; Ziegler, Pech-Georgel, George, \& Lorenzi, 2009; Sakaue, 1999; but see also Robertson, Joanisse, Desroches, \& Ng, 2009). Although the hypothesized nature of these deficits differ in detail, most 
researchers assumed that deficits in auditory processing or speech perception result in (or result from) impoverished phonological representations, which provoke difficulty in learning to read (Boada \& Pennington, 2006). Recently, an alternative viewpoint was suggested that speech perception deficits surface only as a function of stimulus context (e.g., Ahissar, Lubin, Putter-Katz, \& Banai, 2006; Chandrasekaran, Hornickel, Skoe, Nicol, \& Kraus, 2009). Ahissar et al. (2006) observed in their study that individuals with dyslexia did not benefit from repetitive presentation of the same auditory stimulus, and proposed the "perceptual anchor" theory. Similarly, Chandrasekaran et al. (2009) found that dyslexic children exhibited impairments in their ability to modify representation in repetitive stimulus contexts in a speech perception task using auditory brainstem responses. They suggested this impairment may contribute to a deficit in noise-exclusion (Sperling, Lu, Manis, \& Seidenberg, 2005), a hallmark symptom of developmental dyslexia. These researchers encouraged a deeper examination of the dynamic processes in speech perception. In the present study, therefore, we investigated the presence of speech perception in noise deficits that has been repeatedly reported in alphabetic languages and in Japanese children with RD. Specifically, we focused on the context-dependent processes in speech perception in noise, through the effects of 
stimulus presentation rate.

The acquisition of reading is thought to be a culture-specific skill. Indeed, it is assumed that phonological properties and orthographic consistency strongly influence the acquisition of reading (Ziegler \& Goswami, 2006). In the Japanese language, the segmental unit of speech sounds is based on a subsyllabic unit, the mora (Otake, Hatano, Cutler, \& Mehler, 1993). Moreover, due to the constraint that a mora usually consists of a consonant and a vowel (Kubozono, 1999), the amount of required phonological analysis of speech sounds for Japanese listeners is thought to be relatively low. On the other hand, in the Japanese writing system, phonologic and logographic scripts, "Kana" and "Kanji," respectively, are used concurrently. Therefore, in contrast to an alphabetic language such as English, Kana has a highly consistent orthography as each character usually represents one mora. Given that the importance of auditory processing in reading may vary according to orthographic consistency (Georgiou, Protopapas, Papadopoulos, Skaloumbakas, \& Parrila, 2010), these characteristics of Japanese language may influence the relationship between reading and speech perception in Japanese children. Indeed, in an earlier epidemiological study, it was reported that the prevalence of reading problems in Japanese children was very low $(0.98 \%)$ compared to 
those reported in some alphabetic languages (Makita, 1968; see Uno, Wydell, Haruhara, Kaneko, Shinya, 2009 for more details). However, compared to alphabetic languages, few studies exist on nonalphabetic languages of the relationship between reading and speech perception.

Therefore, the present study aimed to address the following questions: (i) Is there evidence for context-dependent processes in speech perception in noise, and does it relate to reading in Japanese children?; (ii) Is there a speech perception in noise deficit in Japanese children with RD, and if so, is it related to context-dependent processes, rather than to the presence of external noise itself?

\section{Method}

\subsection{Participants}

Participants in this study included 117 typically developing (TD) children and 10 children with RD (Table 1). All of them were Japanese natives and recruited from mainstream primary schools. For inclusion, all children with RD possessed difficulty in the acquisition of reading and writing, and had general intelligence levels within normal limits $(>80)$ on a Verbal or Performance IQ estimate from the Japanese version of the Wechsler Intelligence Scale for Children, Third Edition (Japanese WISC-III Publication 
Committee, 1998). The averages were $96.9(S D=11.7)$ and $103(S D=14.7)$ for VIQ and PIQ respectively. They were ongoing participants of our research program, and their reading difficulties had been confirmed in a previous study (Inoue, Higashibara, Okazaki, \& Maekawa, submitted). Exclusion criteria, as determined from parental or teachers' reports, included any history of neurological problems, uncorrected hearing or vision problems, and other developmental disorders such as pervasive developmental disorder or autism.

Prior to enrollment and participation, all participants were informed of the experimental design, and permission to participate was obtained from the parents of children with RD, or from the headmaster of the school who enrolled TD children.

$<$ Insert Table 1 here $>$

\subsection{Materials and Design}

\subsubsection{Reading tasks}

A task to assess reading ability was adopted from a previous study (Inoue et al.,

submitted). Reading ability was assessed by having children read Kana single characters, words, and nonwords. The numbers of each item were 13 respectively, and the length of words and nonwords ranged from three to five characters. The time to read each item 
and the rate of error trials were recorded.

\subsubsection{Phonological processing tasks}

Two tasks adopted from previous research (Inoue et al., submitted) were used to assess phonological skills. In the mora deletion task, children had to delete a certain mora $(/ \mathrm{ta} /)$ of a spoken word and pronounce the remaining part of the word. The position of the mora to be removed varied across items. Response times for each item and error rates were recorded. In the nonword repetition task, children had to repeat aloud a nonword, and error rates were recorded. The numbers of each item were 13 respectively, and the length of words and nonwords ranged from three to five morae. In these tasks, stimuli were presented to the children by a laptop computer via headphones (audio-technica ATH-PRO5V).

\subsubsection{Standardized tests}

General language abilities in children with RD were assessed using the Vocabulary and Similarity subtest scores from the WISC-III. These measures are part of this test's VIQ component. In cases that these tests had already administered within a year, those scores were used for analysis.

\subsubsection{Speech perception in noise task}


In the speech perception in noise task, two monosyllabic sounds /ba/ and /da/ taken from a speech test soundtrack (TY-89) and recorded by Yonemoto (1995) [Fig. 1] were used. Multi-talker noise taken from the same soundtrack was added to the speech sounds at $0 \mathrm{~dB}(\mathrm{RMS})$ signal-to-noise ratio (SNR). Utterance of the speech sounds occurred $200 \mathrm{~ms}$ after a preceding noise stimulus. The noise had $50 \mathrm{~ms}$ rise/fall times. The duration of the speech sounds ranged from 160 to $181 \mathrm{~ms}$.

To vary the stimulus context, two experimental conditions were tested. First, in the same rate condition, each stimulus was presented with equal frequency. In the high rate condition, one stimulus was presented more frequently $(p=0.75)$ than another. In addition, a control condition was utilized in which each stimulus was presented with equal frequency in silence. Each stimulus was presented binaurally to the children through headphones, and the overall levels were calibrated to produce the most comfortable level (MCL).

$$
<\text { Insert Figure } 1 \text { here }>
$$

\subsection{Procedure}

The children tested individually and without feedback. In each condition, there were 20 trials and the monosyllabic stimuli were presented randomly with a stimulus 
onset asynchlony of $3 \mathrm{sec}$. All children started with the quiet condition. Presentation of the other conditions was counterbalanced. The children were asked to identify each syllable and respond by pressing the requisite keyboard buttons. Responses in each condition were measured by reaction time (RT) for speed, coefficient of variance (CV) of RT for stability, and error rate (ER) for accuracy. Test-retest reliabilities with a sub-sample of TD children $(\mathrm{n}=20)$ were $0.71,0.62$, and 0.73 for RT, CV, and ER, respectively.

The tasks were conducted in a quiet room at a university or at the children's schools, and divided into two sessions completed in approximately 30 minutes including breaks. The order of the tasks was counterbalanced.

\section{Results}

\subsection{Speech perception performance in TD children}

Descriptive statistics for all variables in the TD group are shown in Table 2. Two-way analysis of variance (ANOVA) with grade and condition as independent variables revealed main effects for grade on RT, CV, and ER [RT: $F(5,111)=10.41, p<$ 0.001; CV: $F(5,111)=7.30, p<0.001$; ER: $F(5,111)=6.40, p<0.001]$. All variables 
revealed developmental change. There was also a main effect for condition on $\mathrm{CV}[F(1$, 111) $=4.92, p<0.05]$. Responses in the high rate condition were significantly more stable than those in other conditions. Thus, the facilitative effect of stimulus presentation context was confirmed. No interaction between grade and condition was found [RT: $F(5,111)=0.46, p=0.81$; CV: $F(5,111)=1.28, p=0.28$; $\mathrm{ER}: F(5,111)=$ $1.34, p=0.25]$.

\subsection{Correlation and regression analyses}

To explore the relationships among speech perception, reading, and phonological processing in TD children, partial correlations controlling for age and for both age and performance in the quiet condition, were calculated. These correlations are presented in Table 3. When controlling for age, significant correlations were observed among certain variables in each condition of speech perception, reading, and mora deletion. However, when controlling for both age and performance in the quiet condition, significant results were observed only between RT and CV in the high rate condition, reading words and nonwords, and mora deletion.

We also conducted fixed-entry multiple regression analyses to predict reading and phonological processing from performance in speech perception in noise (Table 4). 
Reaction times for reading and mora deletions and accuracy for nonword repetition were the dependent variables. The independent variables were entered as follows in a fixed order: (1) age in months, (2) performance in the quiet condition, and (3) performance in the same rate or high rate condition. Results showed that, after controlling for age and performance in the quiet condition, response stability in the high rate condition accounted for a significant amount of variance in reading words $\left(\Delta R^{2}=\right.$ $0.05, p<0.01)$ and nonwords $\left(\Delta R^{2}=0.03, p<0.05\right)$, and mora deletion $\left(\Delta R^{2}=0.05, p<\right.$ 0.01). No variables in the same rate condition explained any statistically significant amounts of variance in any dependent variable.

$<$ Insert Tables 2, 3, and 4 here $>$

\subsection{Speech perception in noise deficits in children with $R D$}

To examine if there is speech perception in noise deficit in children with $\mathrm{RD}$, comparisons between groups were conducted (Fig. 2). Results of Mann-Whitney $U$ tests showed no significant group difference in the quiet condition $(p>0.05)$. Thus, children with RD did not differ from TD children in simple speech identification. As expected from previous studies in alphabetic languages (Ahissar et al., 2006;

Chandrasekaran et al., 2009), ER of children with RD was significantly higher than that 
of TD children in the high rate condition $(z=-2.83, p<0.01)$, but not in the same rate condition $(z=-1.05, p=0.29)$. No other group difference in RT and CV in both conditions reached significance $(p>0.05)$. Results of Wilcoxon signed-rank tests showed that, in all variables, there were no significant differences between conditions in children with RD (RT: $z=-0.46, p=0.65$; CV: $z=-0.15, p=0.88$; ER: $z=-1.61, p=$ 0.11). In contrast to TD children, all performances in the high rate condition were worse than those in the same rate condition in children with RD.

Next, for analyses at the individual level, we plotted scores of each participant for both groups (Fig. 3). Prior to analyses, we converted all variables (i.e., RT, CV, and ER) of each condition into Z-scores relative to grade-matched TD children of each individual, and averaged these Z-scores to produce composite variables for each condition. We also converted reading speed and mora deletion speed into Z-scores. These scores of each individual are shown in Table 5. In the present study, we chose 1.65 SD for the criterion for deviance. Deviance analysis of these scores found that, while almost all participants with $\mathrm{RD}$ exhibited poor performance in reading and mora deletion, a small sub-group of children with $\mathrm{RD}$ (one in the same rate condition and three in the high rate condition) showed speech perception in noise deficits. An 
individual with RD who had the speech perception deficit in the same rate condition also showed significantly low scores on the Vocabulary and Similarity subtests of the WISC-III.

$<$ Insert Figures 2, 3 and Table 5 here $>$

\section{Discussion}

This study aimed to examine the effect of stimulus context on speech perception and its relationship to reading in TD children and children with RD in Japan. Speech perception in noise tasks were administered within two conditions that varied stimulus presentation rate.

\subsection{Context effects on speech perception and its relationship to reading in TD children}

First, we found that TD children exhibited more stable responses in the high rate condition relative to the same rate condition. This result suggests that there was a facilitatory effect of stimulus context on speech perception. In a previous study of stimulus context effect on speech perception using different techniques, it was suggested that listeners may profit from repetitive stimulus context (Chandrasekaran et al., 2009). Those authors proposed that the mechanisms of such context-dependent facilitatory process in speech perception include top-down modulation of representation. 
Our study found similar context-dependent effects on speech perception in noise by using behavioral measures. Because of the absence of an interaction between grade with condition, context effects may not result from reading experience.

In correlation analyses, a subset of variables in the high rate condition significantly correlated with reading and mora deletion after controlling for age and the quiet condition, whereas those in the same rate condition did not. Similarly, in regression analyses, response stability in the high rate condition accounted for a small but unique amount of variance in reading speed and mora deletion speed, after controlling for age and control condition. These results suggest that in TD children, there are relationships among one's ability to modulate speech perception depending on the stimulus context, reading proficiency, and phonological manipulation. More importantly, these measures of speed are thought to be the most important profile components of Japanese children with RD (Inoue et al., submitted). Therefore, deficits in the modulation of speech perception may relate to reading acquisition in Japanese children.

4.2. Deficits in context-dependent processes of speech perception and their relationship to reading difficulties in children with $R D$ 
As a group, children with RD showed significantly lower accuracy than did TD children in the high rate condition, but not in the same rate condition. This result is consistent with findings from previous studies conducted in alphabetic languages (Ahissar et al., 2006; Chandrasekaran et al., 2009), which posits that their speech perception deficits are not only related to the acoustics of stimulus, but are also related to the stimulus context. Our findings were also broadly consistent with the idea that sensory deficits in individuals with RD are a failure of top-down expectancy-based processes (Ramus, Rosen, Dakin, Day, Castellote, White, \& Frith, 2003; Ramus \& Szenkovits, 2008). Because there was no difference between groups in the same rate condition, our findings appear to contradict most studies that yielded group differences in speech perception in noise. Furthermore, an individual with RD who had the speech perception deficit in the same rate condition also showed significantly low scores on the Vocabulary and Similarity subtests of the WISC-III. A possible interpretation of these results is that individuals with $\mathrm{RD}$ who are vulnerable to the addition of noise may also have language impairments (Robertson et al., 2009). Obviously, further studies are needed to examine it.

Although significant group differences were observed, only a small subgroup 
(30\%) of children with RD displayed significant speech perception in noise deficits

while others did not. These results are consistent with some previous studies that

revealed auditory deficits in only some of their participants (Boets et al., 2007; Ramus

et al., 2003). The number of individuals with speech perception deficits in the present

study was relatively low, and this incidence may at least partially reflect the difference

of phonology and orthography between languages (Georgiou et al., 2010). As mentioned above, because of the characteristics of Japanese phonology (Kubozono, 1999; Otake et al., 1993), the requirement of phonological speech analysis is relatively low. Therefore, it is thought that some Japanese children with RD may be capable of representing them appropriately despite their disability.

\subsection{Limitation}

The small number of children with RD in this study limited the power in statistical analyses. As mentioned earlier, the small sample was at least partly due to the rarity of RD in Japan, especially in Kana reading (Makita, 1968; Uno et al., 2009). Moreover, until recently, there was no well-established diagnostic procedure for identifying specific reading disabilities or dyslexia in Japan. This issue demands attention in clinical practice as well as research. A second limitation of the study was the 
difficulty in comparing our findings directly with those of prior research because of variability in experimental procedures. A systematic crosslinguistic study using identical stimulus and methodology is necessary to elucidate the nature of speech perception deficits in RD across languages.

\subsection{Conclusion}

In conclusion, our findings indicated that Japanese children with RD have speech perception in noise deficits. The underlying factors in these deficits relate to the dynamic processes involved, such as stimulus context-dependent modulation, rather than to the presence of external noise itself. However, the link between speech perception and reading in Japanese children appears to be relatively weaker than it does in alphabetic languages such as English. 


\section{References}

Adlard, A. \& Hazan, V. (1998). Speech perception in children with specific reading difficulties (dyslexia). The Quarterly Journal of Experimental Psychology, 51, $153-177$.

Ahissar, M., Lubin, Y., Putter-Katz, H., \& Banai, K. (2006). Dyslexia and the failure to form a perceptual anchor. Nature Neuroscience, 9, 1558-1564.

Banai, K. \& Ahissar, M. (2004). Poor frequency discrimination probes dyslexics with particularly impaired working memory. Audiology and Neuro-Otology, 9, 328-340.

Boada, R. \& Pennington, B. F. (2006). Deficient implicit phonological representations in children with dyslexia. Journal of Experimental Child Psychology, 95, 153-193.

Boets, B., Ghesquière, P., van Wieringen, A., \& Wouters, J. (2007). Speech perception in preschoolers at family risk for dyslexia: Relations with low-level auditory processing and phonological ability. Brain and Language, 101, 19-30.

Bradlaw, A. R., Kraus, N., \& Hayes, E. (2003). Speaking clearly for children with learning disabilities: Sentence perception in noise. Journal of Speech, Language, and Hearing Research, 46, 80-97.

Brady, S., Shankweiler, D., \& Mann, V. (1983). Speech perception and memory coding in relation to reading ability. Journal of Experimental Child Psychology, 35, $345-367$.

Chandrasekaran, B., Hornickel, J., Skoe, E., Nicol, T., \& Kraus, N. (2009).

Context-dependent encoding in the human auditory brainstem relates to hearing speech in noise: Implication for developmental dyslexia. Neuron, 64, 311-319.

Chermak, G. D., Vonhof, M. R., \& Bendel, R. B. (1989). Word identification performance in the presence of competing speech and noise in learning disable 
adults. Ear and Hearing, 10, 90-93.

Georgiou, G. K., Protopapas, A., Papadopoulos, T. C., Skaloumbakas, C., \& Parrila, R. (2010). Auditory temporal processing and dyslexia in an orthographically consistent language. Cortex, 46, 1330-1344.

Goswami, U., Thomson, J., Richardson, U., Stainthorp, R., Hughes, D., Rosen, S., \& Scott, S. K. (2002). Amplitude envelope onsets and developmental dyslexia: A new hypothesis. Proceedings of the National Academy of Science of the United States of America, 99, 10911-10916.

Inoue, T., Higashibara, F., Okazaki, S., \& Maekawa, H. (submitted). Relationship between reading ability and phonological processing in children with reading difficulties: Reading latency and articulation time.

Japanese WISC-III Publication Committee. (1998). Japanese version of Wechsler Intelligence Scale for Children Third Edition. Nihon Bunka Kagakusha, Tokyo. Kubozono, H. (1999). The Sound System of Japanese. Iwanami, Tokyo. (in Japanese). Makita, K. (1968). The rarity of reading disability in Japanese children. American Journal of Orthopsychiatry, 38, 599-614.

McArthur, G. M., Ellis, D., Atkinson, C. M., \& Coltheart, M. (2008). Auditory processing deficits in children with reading and language impairments: Can they (and should they) be treated? Cognition, 107, 946-977.

Otake, T., Hatano, G., Cutler, A., \& Mehler, J. (1993). Mora or syllable? Speech segmentation in Japanese. Journal of Memory and Language, 32, 258-278.

Ramus, F., Rosen, S., Dakin, S. C., Day, B. L., Castellote, J. M., White, S., \& Frith, U. (2003). Theories of developmental dyslexia: Insights from a multiple case study of dyslexic adults. Brain, 126, 841-865.

Ramus, F. \& Szenkovits, G. (2008). What phonological deficit? Quarterly Journal of 
Experimental Psychology, 61, 129-141.

Robertson, E. K., Joanisse, M. F., Desroches, A. S., \& Ng, S. (2009). Categorical speech perception deficits distinguish language and reading impairments in children. Developmental Science, 12(5), 753-767.

Rosen, S. (2003). Auditory processing in dyslexia and specific language impairment: Is there a deficit? What is its nature? Does it explain anything? Journal of Phonetics, $31,509-527$.

Sakaue, K. (1999). Study on auditory perception of children with learning disabilities-About the ability of speech discrimination under the quasi-noise-. Special Education Research: Department of Special Education Osaka Kyoiku University, 22, 61-69 (in Japanese).

Snowling, M. J. (2000). Dyslexia. Blackwell, Oxford, U. K.

Sperling, A. J., Lu, Z.-L., Manis, F. R., \& Seidenberg, M. S. (2005). Deficits in perceptual noise exclusion in developmental dyslexia. Nature Neuroscience, 8 , $862-863$

Tallal, P. (1980). Auditory temporal perception, phonics, and reading disabilities in children. Brain and Language, 9, 189-192.

Uno, A., Wydell, T. N., Haruhara, N., Kaneko, M., \& Shinya, N. (2009). Relationship between reading/writing skills and cognitive abilities among Japanese promary-school children: normal readers versus poor readers (dyslexics). Reading and Writing: An Interdisciplinary Journal, 22, 755-789.

Vellutino, F. R., Fletcher, J. M., Snowling, M. J., \& Scanlon, D. M. (2004). Specific reading disability (dyslexia): what have we learned in the past four decades? Journal of Child Psychology and Psychiatry, 45, 2-40.

Yonemoto, K. (1995). Characteristics of CD (TY-89) for evaluation of hearing-aid 
fitting. Journal of Otolaryngology, Head and Neck Surgery, 11, 1395-1401 (in Japanese).

Ziegler, J. C. \& Goswami, U. (2006). Becoming literate in different languages: Similar problems, different solutions. Developmental Science, 9, 429-453.

Ziegler, J. C., Pech-Georgel, C., George, F., \& Lorenzi, C. (2009).

Speech-perception-in-noise deficits in dyslexia. Developmental Science, 12, $732-745$ 


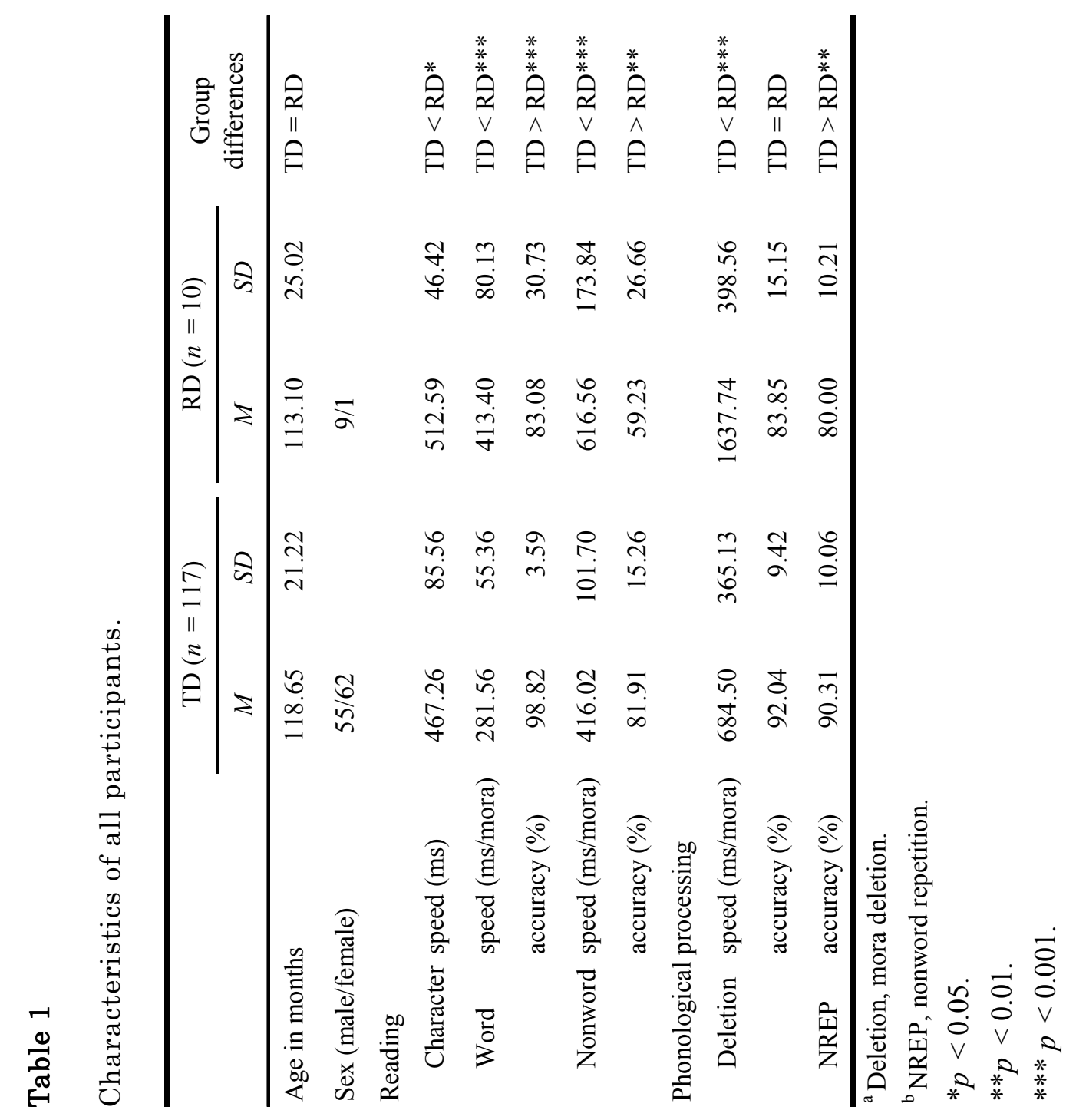


ন






\section{Table 3}

Partial correlations among measures controlling for age (top) and for both age and the quiet condition (bottom) in TD children.

\begin{tabular}{|c|c|c|c|c|c|c|c|c|c|}
\hline & & \multicolumn{5}{|c|}{ Reading } & \multicolumn{3}{|c|}{ Phonological Processing } \\
\hline & & \multirow{2}{*}{$\begin{array}{c}\text { Character } \\
\text { speed }\end{array}$} & \multicolumn{2}{|c|}{ Word } & \multicolumn{2}{|c|}{ Nonword } & \multicolumn{2}{|c|}{ Deletion } & \multirow{2}{*}{$\begin{array}{c}\text { NREP } \\
\text { accuracy }\end{array}$} \\
\hline & & & speed & accuracy & speed & accuracy & speed & accuracy & \\
\hline \multirow[t]{3}{*}{ Same rate } & RT & .13 & .15 & .17 & $.20 *$ & -.09 & .07 & -.11 & -.04 \\
\hline & $\mathrm{CV}$ & -.15 & .10 & -.03 & .09 & -.04 & .16 & -.15 & .07 \\
\hline & ER & $-.25 * *$ & -.01 & -.04 & -.12 & -.04 & .13 & -.16 & -.18 \\
\hline \multirow[t]{3}{*}{ High rate } & RT & $.28 * *$ & $.33 * * *$ & .01 & $.39 * * *$ & -.10 & .14 & .05 & -.03 \\
\hline & $\mathrm{CV}$ & .03 & $.26 * *$ & $-.24 * *$ & $.23 *$ & -.12 & $.31 * * *$ & -.15 & .01 \\
\hline & ER & -.03 & .10 & -.03 & .07 & -.03 & .07 & .00 & .01 \\
\hline \multirow[t]{6}{*}{ Quiet } & $\mathrm{RT}$ & $.23 *$ & $.28 * *$ & .10 & $.36 * * *$ & -.16 & .17 & .02 & .00 \\
\hline & $\mathrm{CV}$ & $-.19 *$ & .01 & -.14 & .07 & -.08 & $.25 * *$ & -.11 & -.10 \\
\hline & ER & $-.22 *$ & -.05 & .01 & -.12 & -.08 & $.23 *$ & -.13 & -.10 \\
\hline & & \multicolumn{5}{|c|}{ Reading } & \multicolumn{3}{|c|}{ Phonological Processing } \\
\hline & & \multirow{2}{*}{$\begin{array}{c}\text { Character } \\
\text { speed }\end{array}$} & \multicolumn{2}{|c|}{ Word } & \multicolumn{2}{|c|}{ Nonword } & \multicolumn{2}{|c|}{ Deletion } & NREP \\
\hline & & & speed & accuracy & speed & accuracy & speed & accuracy & accuracy \\
\hline \multirow[t]{3}{*}{ Same rate } & RT & -.01 & -.01 & .14 & .00 & -.00 & -.04 & -.15 & -.05 \\
\hline & $\mathrm{CV}$ & -.10 & .10 & .02 & .08 & -.02 & .10 & -.12 & .11 \\
\hline & ER & -.18 & .01 & -.05 & -.08 & -.02 & .06 & -.12 & -.16 \\
\hline \multirow[t]{3}{*}{ High rate } & RT & .18 & $.21 *$ & -.06 & $.24 * *$ & -.01 & .04 & .04 & -.04 \\
\hline & $\mathrm{CV}$ & .07 & $.26^{* *}$ & $-.22 *$ & $.22 *$ & -.11 & $.27 * *$ & -.13 & .03 \\
\hline & ER & .03 & .12 & -.03 & .10 & -.01 & .02 & .03 & .03 \\
\hline
\end{tabular}

${ }^{\mathrm{a}}$ Deletion, mora deletion.

${ }^{\mathrm{b}}$ NREP, nonword repetition.

${ }^{*} p<0.05$.

$* * p<0.01$.

$* * * p<0.001$. 


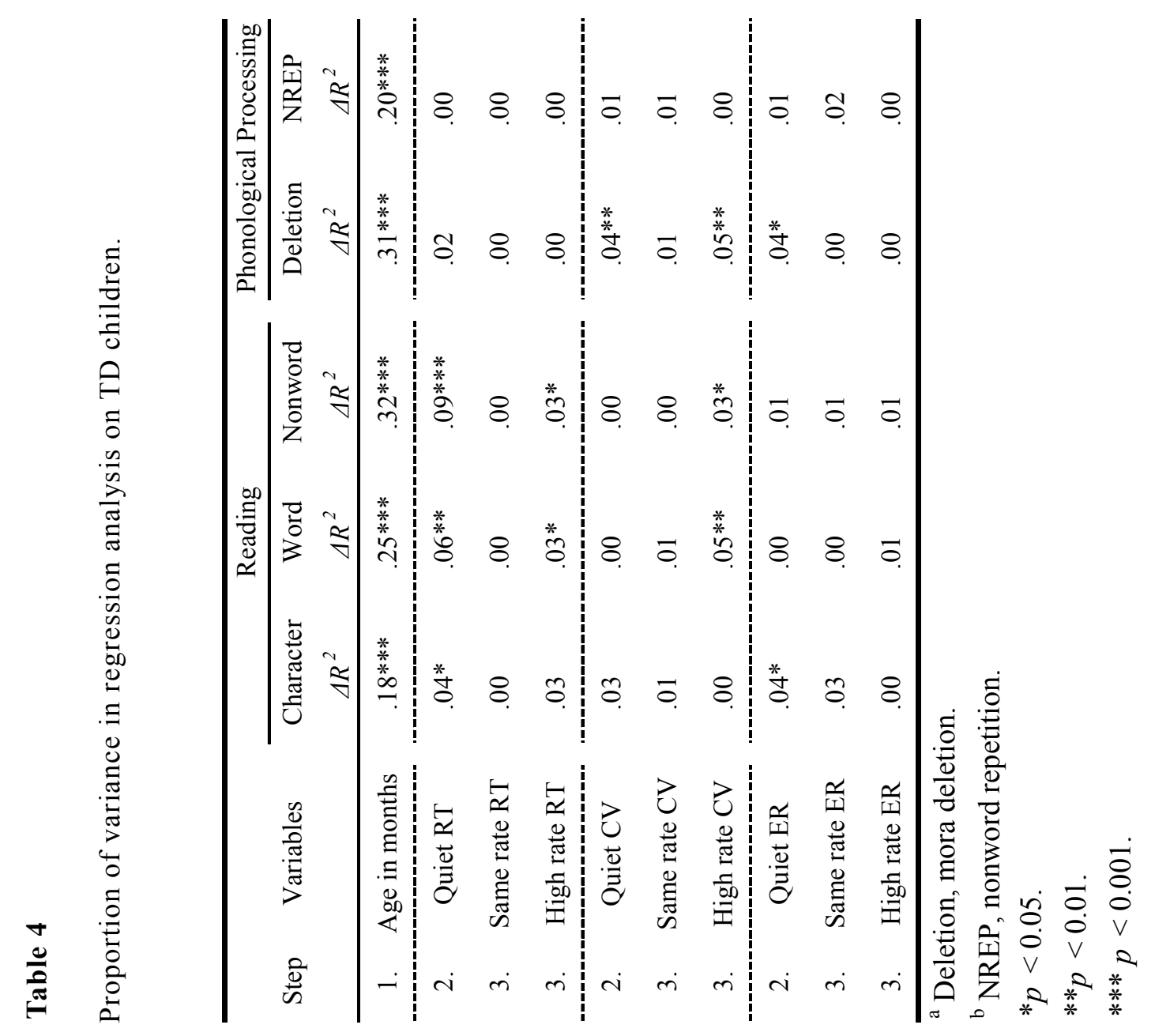




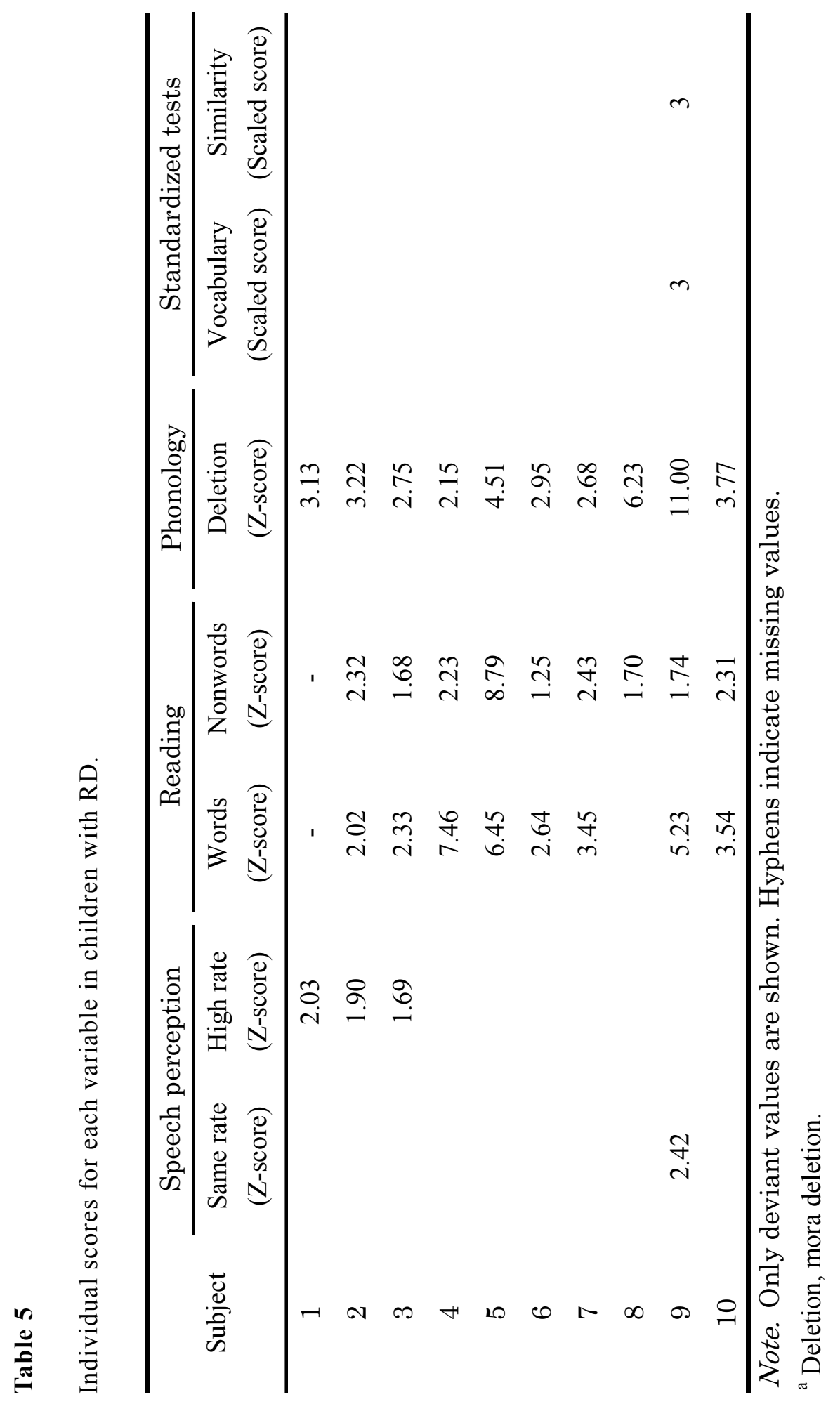


(a)
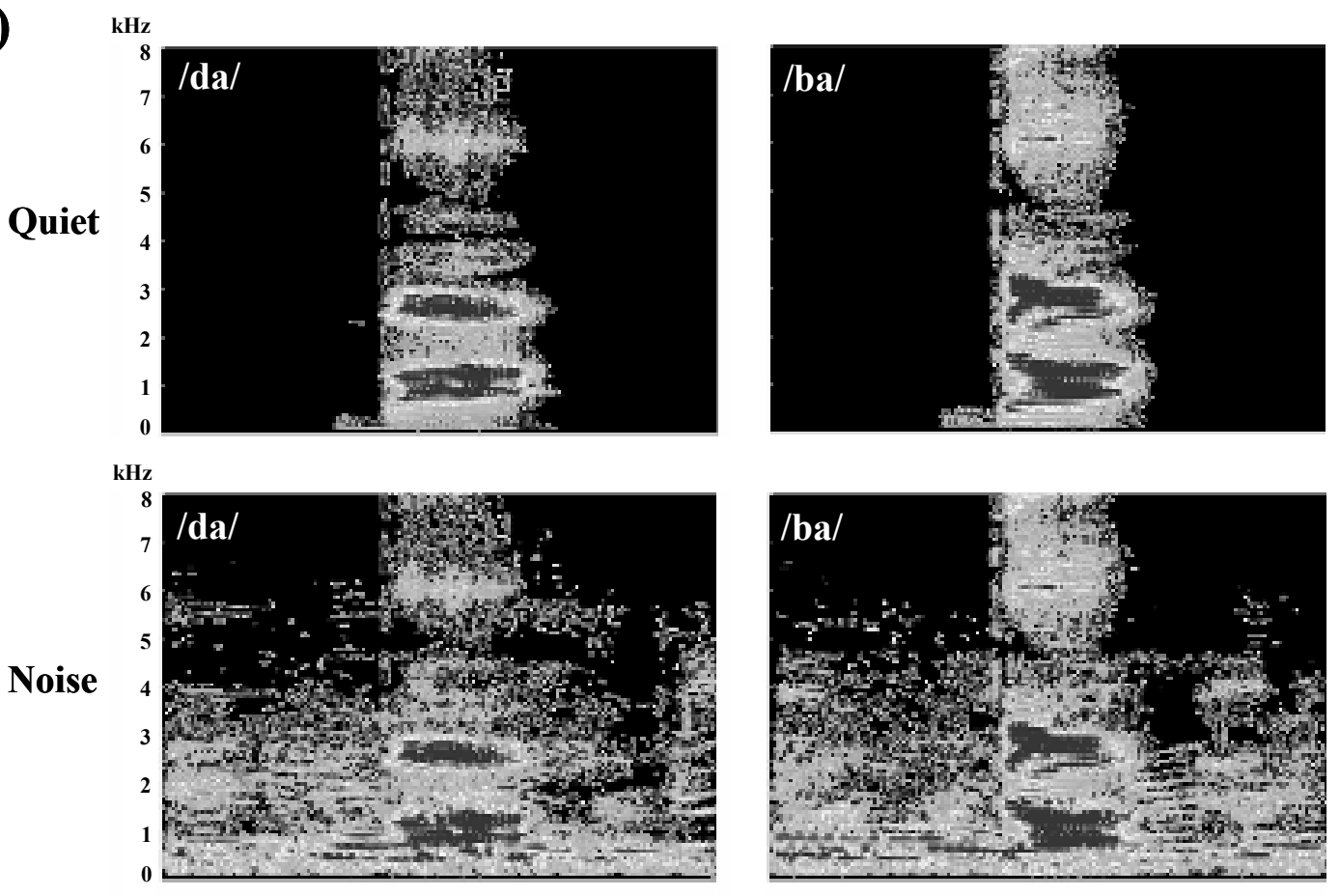

(b)

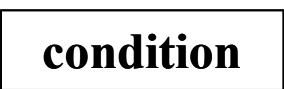

\section{stimulus}

$\begin{array}{ll}\text { Same rate } & / \mathrm{da} /-/ \mathrm{da} /-\underline{\mathrm{ba} /}-/ \mathrm{da} /-\underline{\mathrm{ba} /}-\underline{\mathrm{ba} /} \\ \text { High rate } & \underline{\mathrm{ba} /}-\underline{\mathrm{ba} /}-\underline{\mathrm{ba} /}-\underline{\mathrm{ba} /}-/ \mathrm{da} /-\underline{\mathrm{ba} /}\end{array}$

Fig. 1. Sound spectrograms of speech stimulus (a) and schematics of stimulus presentation conditions (b). 

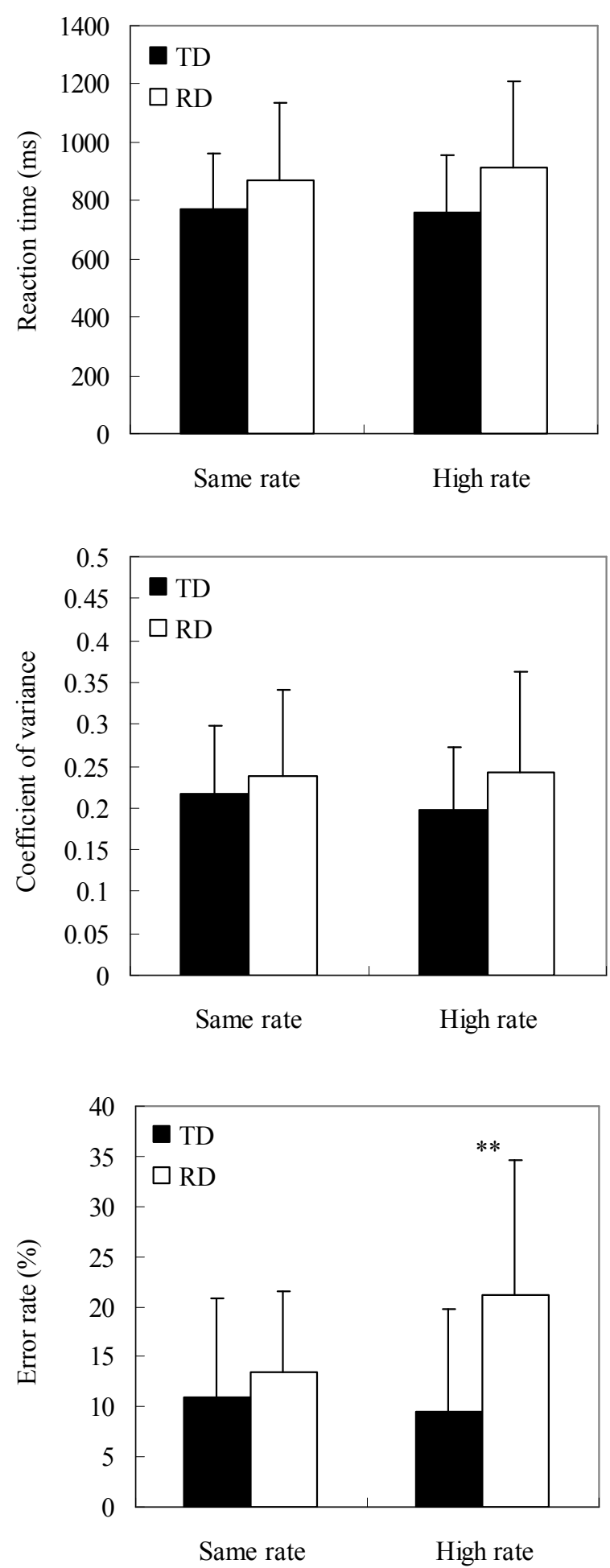

Fig. 2. Performances of speech perception in noise in TD children as well as those with RD. Error bars indicate plus one standard deviation of the mean per group. 



Fig. 3. Individual Z-scores on reading, phonological processing, and speech perception in noise. To summarize reading words and nonwords, these two Z-scores were averaged. The solid line indicates the average of TD children. The dashed line indicates the chosen deviance criterion (1.65 SD above the average of TD children). Deviant individuals are identified. 